\title{
Traumatic anterior hip dislocation associated with anterior and inferior iliac spines avulsions and a capsular-labral lesion
}

\author{
A. Reggiori · G. Brugo
}

Received: 27 January 2008/Accepted: 13 February 2008/Published online: 5 March 2008

(C) Springer-Verlag 2008

\begin{abstract}
A 16-year-old man had a traumatic accident during motor-cross racing and sustained an anterior hip dislocation with fracture of anterior superior and anterior inferior iliac spines. The hip was emergently reduced and further imaging was obtained to evaluate the lesion and bony fragments. Computed tomography confirmed the presence of two large fragments and a small fragment from the antero-inferior acetabulum. The patient underwent open reduction and internal fixation of the iliac spines and the reparation of anterior hip capsule. No complications occur after 1 year of follow-up. We present the case and a review of the literature for this lesion.
\end{abstract}

Keywords Hip fracture · Hip dislocation · Avulsion · Capsular lesion

\section{Introduction}

In high-energy injuries from road-traffic accidents or sports, posterior hip dislocation is more common than the anterior [1]. Fracture dislocation of the hip was first reported by Astley and Cooper in 1791 [2] and classified by Stewart and Milford in 1954 [3].

A review of the orthopaedic literature identifies the reports on isolated avulsions of the anterior superior iliac spine (ASIS), anterior inferior iliac spine (AIIS), ischial tuberosity, iliac apophysis, or reflected head of the rectus femoris in children due to failure of the apophyseal

A. Reggiori $(\bowtie) \cdot$ G. Brugo

Orthopaedic and Trauma Department,

Ospedale Santissima Trinità, Viale Zoppis 10,

28021 Borgomanero, Novara, Italy

e-mail: andreareggiori@yahoo.it cartilage but such associated injuries are uncommon in adults [4]. The classification system by Tile [5] recognized avulsions of the iliac spines, crest and ischial tuberosity as well as iliac wing fractures but it is rare for fracture dislocations of the hip to involve these bony sites.

We describe a case with an anterior fracture dislocation of the hip with several associated bony injuries and a capsular-labral lesion.

\section{Case report}

A 16-year-old man presented in our hospital with right hip and lower extremity pain following a motor-cross racing accident. The patient was conscious and alert, well oriented with stable vital signs on arrival. He complained only of right hip pain, and physical examination showed an externally rotated and short right leg. $\mathrm{He}$ was free of neurovascular impairment. Plain radiographs showed an anterior dislocation of the right hip with two large and two smaller bony fragments (Fig. 1a). The hip was reduced under conscious sedation (Fig. 1b) and reduction controlled with skin traction. Further imaging studies, including new radiographs of the hip and computed tomography scans with three-dimensional reconstruction, were obtained. The fracture involved the ASIS and the AIIS at the level of origin of the reflected head of rectus femoris with a small fragment close by. In spite of the hip dislocation, the acetabulum appeared intact in the weightbearing area but a CT image showed a small fragment originating from the inferior part of acetabulum (Fig. 2). The patient underwent surgery 1 week after trauma. During exposure through an ilio-inguinal approach, the lateral femoral cutaneous nerve was identified and mobilized from 
Fig. 1 a Radiograph of the anterior dislocation of the right hip with fracture fragments widely displaced. b X-ray control after closed reduction of the hip
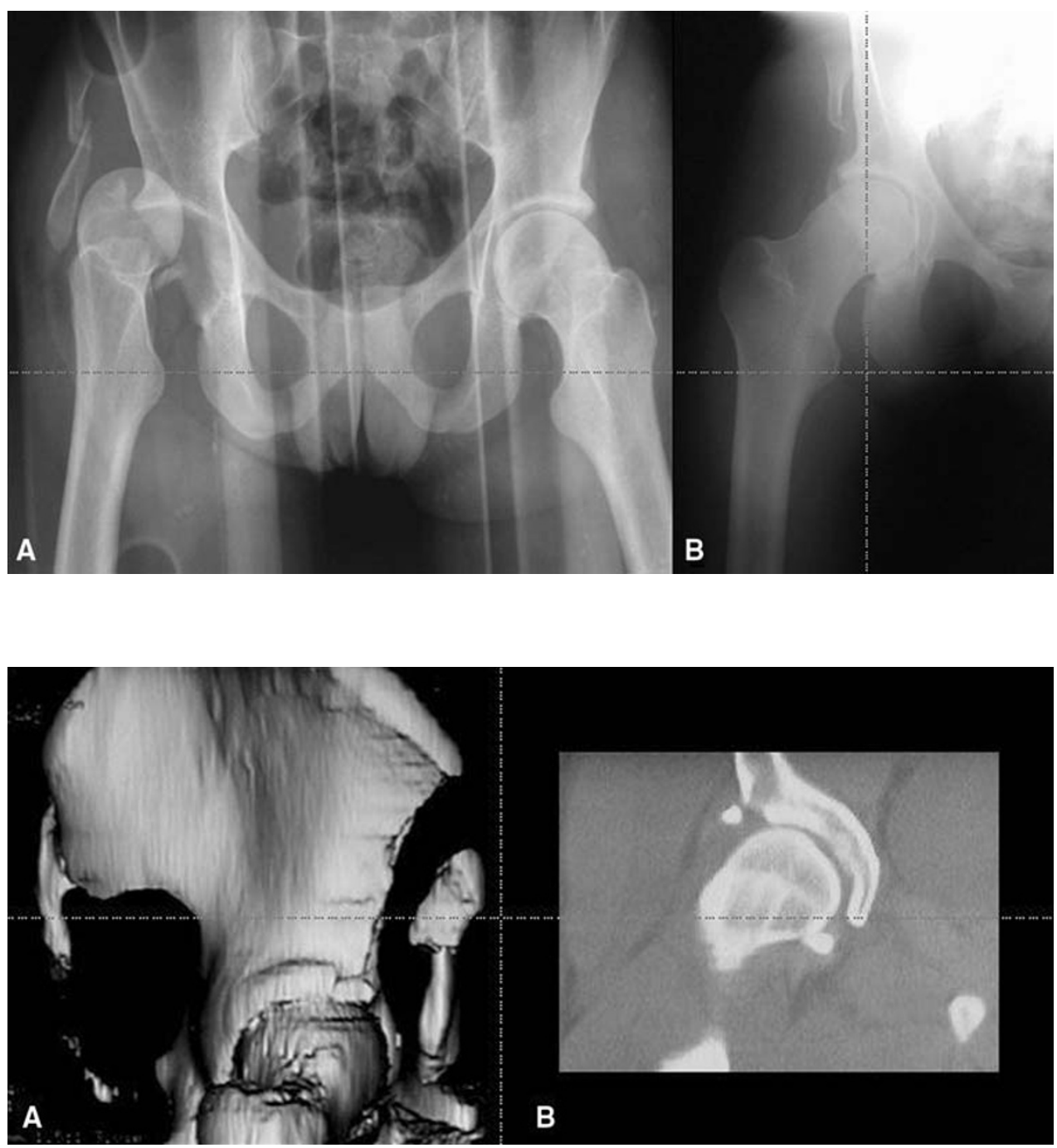

Fig. 2 a Post-reduction 3D CT scan shows the fracture with two big fragments involving ASIS and AIIS. b CT scan shows two small fragments; one is the reflected head of rectus femoris and the other is in the inferior part of acetabulum the fracture site. The nerve appeared intact in the zone of injury and was visually monitored during the procedure. Open reduction of the AIIS fragment (straight head of the rectus femoris) was accomplished and provisionally held with a Kirschner wire. We also noted a bony avulsion of the reflected head of the rectus femoris, a laceration of the anterior capsule with detachment from the acetabular rim and an avulsion of anterior acetabular labrum from articular cartilage. This finding was similar to the Bankart lesion of traumatic shoulder instability. Repair was performed without hip dislocation using an anchor (SuperQuick anchor ${ }^{\mathrm{TM}}$ Plus, DePuy Mitek J\&J) that reattached the acetabular labrum to the edge of the acetabulum. Using the same suture, a repair of the anterior capsule was also performed. Finally, the AIIS bone fragment was fixed with two $3.5 \mathrm{~mm}$ cannulated titanium screws (Hit Medica, Italy). The ASIS fragment that represented the origin of sartorius was isolated, reduced and definitively fixed with a 5-mm cannulated titanium screw (Hit Medica, Italy). It was not possible to gain access and reduce the inferior wall acetabulum fragment using the ilio-inguinal approach. It was decided that the fragment was left in situ after deliberation over its size and location away from the weightbearing portion of the acetabulum. Intraoperative and radiographic checks confirmed excellent reduction of the fragments. Postoperatively, the patient was maintained in a pelvic spica cast in $30^{\circ}$ of hip flexion for 3 weeks to reduce muscle tension on surgical repair sites. Active hip flexion and extension to recover hip and knee movement were then permitted but without weight bearing for 2 months to reduce the risk of femoral head necrosis after dislocation.

Anti-thromboembolic therapy was started after injury using 4,000 IU of low molecular weight Heparin daily until weight bearing. Antibiotics were used during the perioperative period with Teicoplanin $400 \mathrm{mg}$ daily for 2 days. No complications occur during the in-patient stay or the out-patient follow-up. At the time of discharge, the patient was in good condition. 


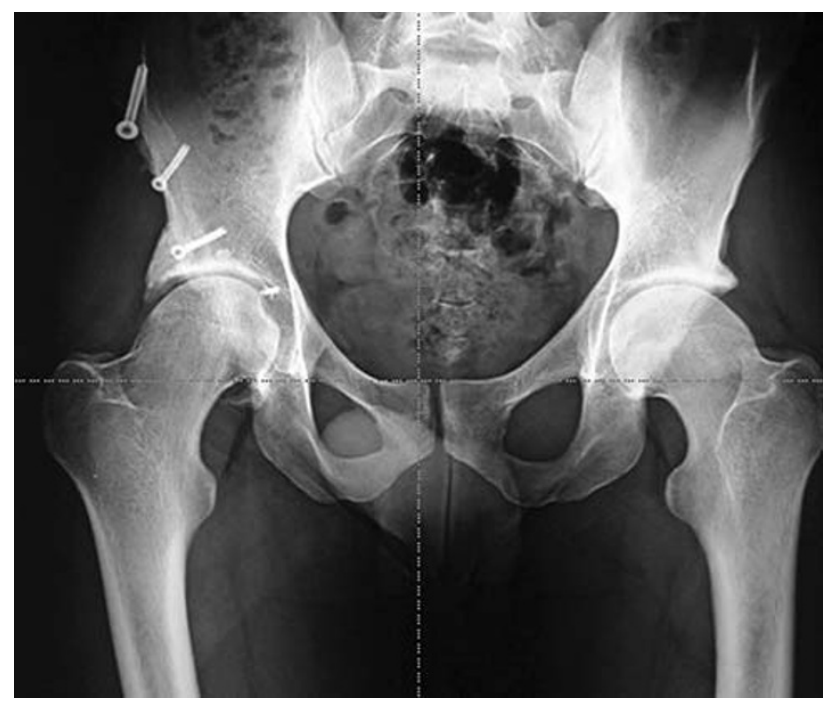

Fig. 3 Pelvic radiograph after 12 months shows bone healing with normal joint space and without heterotopic ossification

Radiographs at 1 year after surgery showed excellent bony healing (Fig. 3). MRI arthrography showed the absence of ulceration or irregularities on the surface of the femoral head or acetabular cartilage. The capsular repair remained intact without evidence of a labral tear and leakage of contrast medium (Fig. 4). The patient was free of pain with a complete range of hip motion and had returned to previous activities including sports and motor-cross racing.

\section{Discussion}

Pelvic trauma may be classified according to the severity of the associated injuries; these include avulsions or epiphyseal separations (secondary to violent muscular activity), pelvic ring fractures (secondary to crush injuries), or acetabular fractures (with or without hip dislocations).

Traumatic hip dislocations are serious injuries because the hip joint is extremely stable and a considerable force is necessary to produce a dislocation. The posterior dislocation of the hip is by far the most common type with a reported ratio of anterior to posterior dislocations ranging from 1:10 to 1:19 [1-6]. Jacob et al. [7] reported 73 posterior, 9 central, and 2 anterior dislocations of the 84 cases. Anterior dislocations are usually caused by a fall from a height producing a direct posterior blow on an abducted externally rotated thigh. Traumatic hip dislocations occur in the third decade of life in about $35 \%$ of the cases with $75 \%$ of the injuries occurring in males [8].

Avulsion injuries of the pelvis occur usually in athletic adolescents. These are divided into avulsions of the ASIS, AIIS, ischial tuberosity, iliac crest, or reflected head of the rectus femoris $[9,10]$. Normally the AIIS and ASIS avulsions are caused by sudden forceful muscular traction on the pelvis by rectus femoris, sartorius, or tensor fascia lata with the hip extended and the knee flexed, while ischial tuberosity fracture avulsions are caused by sudden contraction of the hamstrings or adductors during hip flexion. Although repetitive stress may lead to these avulsions, it is more usual to see these injuries in athletes in whom there is a high likelihood of forceful muscle contraction in stretch [10]. Fracture of the ASIS is also a known complication after harvesting iliac crest bone graft [11].

In this case report, the mechanism of injury was possibly a violent hyperextension of the hip, resulting in the avulsion of the AIIS and ASIS, followed by anterior dislocation of the hip. The diagnosis was established following clinical evaluation of the leg and the pelvic and hip radiographs; CT and MRI reveal more detail but only after the reduction.
Fig. 4 MRI-arthrography images after 12 months show no ulceration or irregularity on the femoral head surface or femoral head osteonecrosis. The capsule is contiguous with no labral tear or leakage of contrast medium

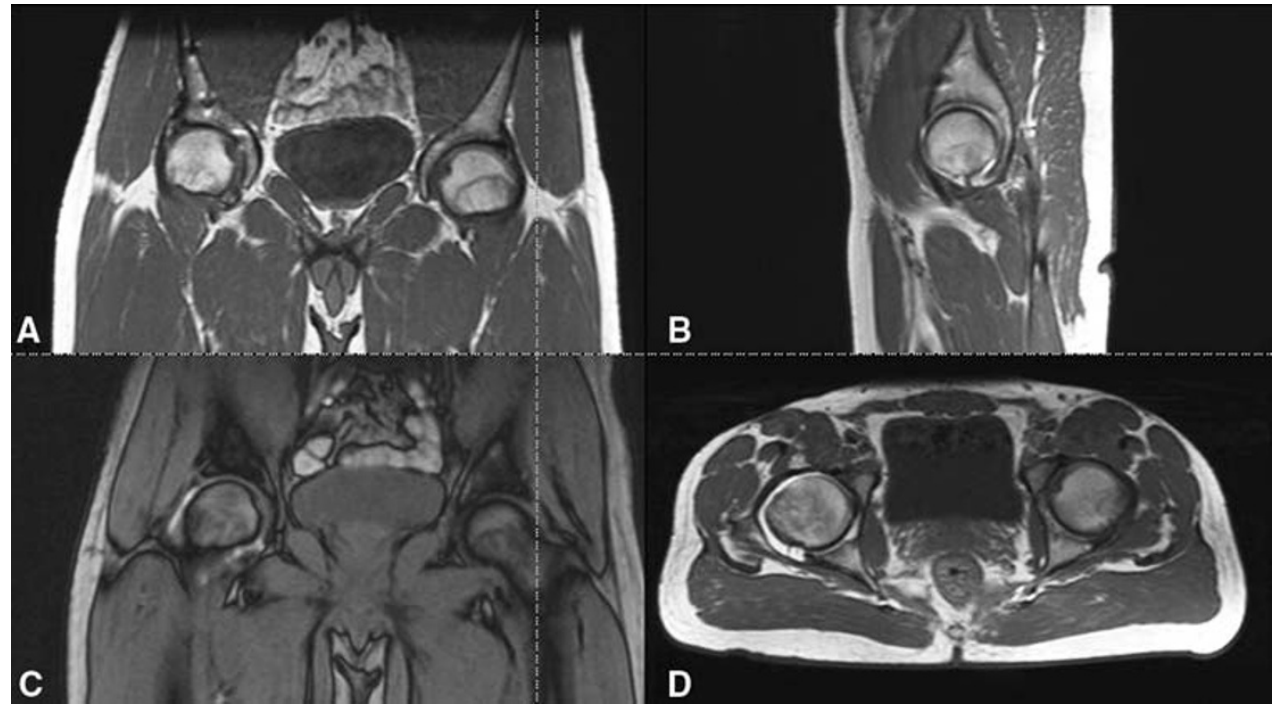


Most authors advocate non-operative treatment of ASIS and AIIS avulsions because the bone fragments are typically small and displaced laterally and inferiorly from ilium. When displacement of these fragments is small, healing occurs with callus formation evident and is associated with a satisfactory functional recovery and return to sports [12, 13]. Some orthopaedists rely solely on nonoperative treatment, whereas others advocate open reduction and internal fixation for certain patients [1, 12-14]. Open reduction and internal fixation are mandatory when the pelvic avulsion injury has significant fragment displacement of more than $3 \mathrm{~cm}$ or if anatomic reduction contributes to restoration of the articular surface, hip joint congruity and stability. It is sometimes considered whether patients require a short convalescent period to avoid protracted bed rest or immobility.

The initial treatment for a patient with hip dislocation is gentle and prompt reduction within $6 \mathrm{~h}$ [15] and preferably under general anaesthesia to prevent and minimize complications [1, 6-8]. An important complication following traumatic dislocation of the hip is prolonged and irreversible ischemia of the head of the femur leading to osteonecrosis in 10-30\% or greater particularly if the dislocation is accompanied by severe bone destruction. The objective of treatment is to obtain an anatomic reduction with congruous hip joint surfaces. Instability, loose fragment retention in the hip joint or incomplete reduction precludes a good result. Sahin et al. considered fragments outside the immediate weight-bearing zone of the hip to be important also; their study reported satisfactory results, were obtained with a prompt and stable hip reduction regardless of the treatment method $[1,16]$.

Surgical treatment can restore anatomy and the attendant biomechanics of the hip joint. This also caters for return of the best muscle control and reduces immobilization time. Possible complications specific to open reduction and internal fixation of the AIIS or ASIS include transection injury to the lateral femoral cutaneous nerve, meralgia paraesthetica, intra-articular screw penetration, infection, and blood loss [17].

In non-operative treatment of pelvic avulsion injuries, the most complication reported in the literature is heterotopic bone formation which sometimes can result in impaired hip joint function, pain and swelling in the inguinal area of sufficient severity to require surgical removal. Other complications of conservative treatment include chronic avulsion with bony bridge formation, nonunion, and persistent pain. A literature review found $68 \%$ of conservatively treated ASIS, AIIS, and ischial tuberosity avulsions did not unite, although good clinical results were achieved [18, 19].

McCarthy et al. [20] reported that acetabular labral tears are generally secondary to trauma and are isolated to one particular region depending on the direction, strength and extension of trauma. Direct trauma, including motor vehicle accidents and falls with or without dislocation, is a recognized cause of labral tears. The magnitude of force will determine whether the tear is initiated on the articular or acetabular side of the joint and sometimes creates an associated capsular lesion. Normally, the treatment of the labral lesions causing a femoral-acetabular impingement syndrome is arthroscopic but some surgeons will undertake an open repair if the lesion is associated with the dislocation of the hip joint [21]. Exploration of the anterior hip joint in our patient gave us the opportunity to diagnose the capsular detachment and labral tear and undertake repair with a small anchor suture.

It has not been established but undiagnosed labral lesions after hip dislocations may be a cause of persistent symptoms in the presence of normal X-rays. It is convenient to link this possibility to the development of posttraumatic osteoarthritis (another fairly common complication of traumatic dislocation of the hip [22]) but again this is not scientifically established.

\section{Conclusion}

There is no consensus regarding therapy after reduction of traumatic hip dislocations. In the past, traction or prolonged immobilization followed by a long period of nonweight-bearing was recommended. Recent reports have not shown deleterious effects from early mobilization, and several authors have documented that a prolonged nonweight-bearing period does not significantly influence the subsequent development of femoral head osteonecrosis [1, 15, 16, 23].

Patients with traumatic hip dislocations associated with bony and capsular lesions will need to have treatment individualized. This case report highlights this strategy for correct decision making and emphasizes a preoperative plan, derived from imaging studies, is imperative for surgery.

\section{References}

1. Sahin V, Karaks ES; Aksu S, Atlihan D et al (2003) Traumatic dislocation and fracture-dislocation of the hip: a long term follow-up study. J Trauma 54:520-529

2. Reigstad A (1980) Traumatic dislocation of the hip. J Trauma 20:603

3. Stewart MJ, Milford LW (1954) Fracture-dislocation of the hip: an end-result study. J Bone Joint Surg Am 36:315

4. Meyer NJ, Schwab JP, Orton D (2001) Traumatic unilateral avulsion of the anterior superior and inferior iliac spines with anterior dislocation of the hip: a case report. J Orthop Trauma 15(2):137-140 
5. Tile M (1988) Pelvic ring fractures: should they be fixed? J Bone Joint Surg [Br] 70:1-12

6. Brav EA (1962) Traumatic dislocation of the hip: army experience and results over a 12-year period. J Bone Joint Surg Am 44:1115

7. Jacob JR, Rao JP, Ciccarell C (1987) Traumatic dislocation and fracture-dislocation of the hip: a long-term follow-up study. Clin Orthop Relat Res 214:249-263

8. Whitehouse GH (1978) Radiological aspects of posterior dislocation of the hip. Clin Radiol 29:431-441

9. Deehan DJ, Beattie TF, Knight D et al (1992) Avulsion fracture of the straight and reflected heads of the rectus femoris. Arch Emerg Med 9:310-313

10. Aksoy B, Ozturk K, Ensenyel CZ et al (1998) Avulsion of the iliac crest apophysis. Int J Sports Med 19:76-78

11. Hu RW, Bohlman HH (1994) Fracture at the iliac bone graft harvest site after fusion of the spine. Clin Orthop 309:208-213

12. Fernbach SK, Wilkinson RH (1981) Avulsion injuries of the pelvis and proximal femur. Am J Roentgenol 137:581-584

13. Sundar M, Carty H (1994) Avulsion fractures of the pelvis in children: a report of 32 fractures and their outcome. Skeletal Radiol 23:85-90

14. Canale ST, King RE (1991) Pelvic and hip fractures. In: Rockwood CA, Wilkins KE, Richard EK (eds) Fractures in children, vol 3. JB Lippincott, Philadelphia, pp 991-1120
15. Hougaard K, Thomsen PB (1986) Traumatic posterior dislocation of the hip: prognostic factors influencing the incidence of avascular necrosis of the femoral head. Arch Orthop Trauma Surg 106:32-35

16. Jaskulka RA, Fischer G, Fenzl G (1991) Dislocation and fracturedislocation of the hip. J Bone Joint Surg Br 73:465-469

17. Saluan PM, Weiker GG (1997) Avulsion of the anterior inferior iliac spine. Orthopaedics 20:558-559

18. Watts HG (1976) Fractures of the pelvis in children. Orthop Clin North Am 7:615-624

19. Irving MH (1964) Exostosis formation after traumatic avulsion of the anterior inferior iliac spine: report of two cases. J Bone Joint Surg [Br] 46:720-722

20. McCarthy J, Noble P, Aluisio FV et al (2003) Anatomy, pathologic features, and treatment of acetabular labral tears. Clin Orthop Relat Res 406:38-47

21. Ganz R, Parvizi J, Beckel M et al (2003) Femoro-acetabular impingement: a cause for osteoarthritis of the hip. Clin Orthop Relat Res 417:112-120

22. Upadhyay SS, Moulton A (1981) The long-term results of traumatic posterior dislocation of the hip. J Bone Joint Surg $\mathrm{Br}$ 63:548-551

23. Schlickewei W, Elsässer B, Mullaji AB et al (1993) Hip dislocation without fracture: traction or mobilization after reduction? Injury $24: 27-31$ 